\title{
Influence of Hindlimb Unweighting and Intermittent Weight Bearing on Dynamics of Nuclei in Rat Soleus Muscle
}

\author{
Toshiaki YAMAZAKI ${ }^{1}$ \\ ${ }^{1}$ Department of Physical Therapy, School of Health Sciences, Faculty of Medicine, Kanazawa University, 5-11-80 Kodatsuno, \\ Kanazawa 920-0942, Japan
}

\begin{abstract}
The purpose of this study was to examine differences in the inhibitory effect of disuse atrophy as a result of intermittent weight bearing in terms of the dynamics of nuclei in rat soleus muscle. Disuse muscle atrophy was induced by hindlimb suspension for two weeks. Forty-nine male Wistar rats (body weight: 190-228 g) were divided into the control group (CON) and the experimental group. The experimental group was subdivided into four groups: hindlimb suspension alone (HS), weight bearing for 10 minutes $\times 4$ times/day (W10), weight bearing for 20 minutes $\times 2$ times/day (W20), and weight bearing for 40 minutes $\times$ one time/day (W40). In addition to histochemical examination, this study examined both cell proliferation and apoptosis in terms of the dynamics of myonuclei immuno-histochemically. The mean cross-sectional area of muscle fibers demonstrated the effect of weight bearing. The number of proliferating myonuclei per 100 muscle fibers was decreased in the experimental groups as compared with CON. Proliferating myonuclei in W10 and W40 were more than HS, indicating the effect of weight bearing. Apoptotic myonuclei was increased in the experimental groups as compared with CON. This parameter in W10 and W40 were statistically not significantly different from CON, suggesting that these weight bearing methods can prevent the loss of myonuclei by apoptosis. However, W20 was not significantly different from HS in terms of the dynamics of myonuclei. This suggests that weight bearing for W20 was ineffective. The results in this study indicated the possibility of inducing different effects by the frequency of weight bearing.

Key words: intermittent weight bearing, myonuclei, hindlimb unweighting, atrophy prevention, soleus muscle
\end{abstract}

(J Jpn Phys Ther Assoc 6: 1-8, 2003)

$\mathrm{T}_{\mathrm{h}}$ disuse muscle atrophy is regarded as an important problem
in physical therapy. For muscle atrophy caused by
unweighting (non-weight bearing) in the lower extremities,
weight bearing is an important factor ${ }^{12}$ ). However, there
are many clinical situations in which it is difficult to impose
weight bearing for a long time. Although there are many
reports ${ }^{3) 4}$ about pathological changes in muscle when
weight bearing is resumed after a period of unweighting,
the clinical situation is more complex because of the need to
alternate weight bearing and unweighting.

Received: March 27, 2002

Accepted: June 29, 2002

Correspondence to: Toshiaki Yamazaki, Department of Physical Therapy, School of Health Sciences, Faculty of Medicine, Kanazawa University, 5-11-80 Kodatsuno, Kanazawa 920-0942, Japan
Some studies have reported the effect of intermittent weight bearing on skeletal muscle during disuse atrophy in animal experiment ${ }^{5-12)}$. Intermittent weight bearing was carried out for $40 \sim 120 \mathrm{minutes} / \mathrm{day}$ at one time or sometimes for 10 15 minutes/day. We have also examined weight bearing effect of one hour/day about frequency, and reported that differences in sensitivity based on fiber type and the interval of weight bearing are important factors $^{2) 13-17)}$.

Recently, some researchers have begun to address issues regarding the role of myonuclei in skeletal muscle adaptation, focusing on the dynamics of myonuclei ${ }^{18)}$. Hypertrophy is known to be accompanied by an increase in both fiber size and myonuclear numbers ${ }^{19)}$. Studies on adult animals undergoing functional overload have supported the view that a satellite cell inducing increase in myonuclear numbers is a necessary component of hypertrophic growth 
in adult muscle ${ }^{18)}$. However, the role of myonuclear modulation during skeletal muscle atrophy in adult animals is not well known. A decrease in myonuclear numbers in atrophying muscles has been reported under certain experimental conditions. These studies have demonstrated that muscle atrophy is associated with a decrease in myonuclear numbers, which may be at least partially responsible for a decrease in fiber size ${ }^{18)}$. Schultz et al. ${ }^{20)}$ reported that a rapid decrease in proliferative activity of satellite cells occurs during the very earliest phases (12 24 hours) of hindlimb unweighting and precedes morphological changes in muscle fibers by 3 days. They further suggested that proliferative activity of satellite cells after initiation of hindlimb unweighting is a sensitive and valid indicator of atrophic changes in growing myofibers and occurs in parallel with early changes in gene expression. Although the mechanism(s) responsible for the loss of myonuclei during muscle atrophy are currently not well defined, several possibilities can be envisioned. The most likely possibility is that myonuclei are eliminated from atrophying adult myofibers by apoptosis ${ }^{18)}$. Allen $e t$ $a l .{ }^{21)}$ reported a reduction in both myonuclear numbers and size of fibers expressing type I myosin heavy chain after 14 days of spaceflight, and suggested the possibility of myonuclear elimination by a modified form of apoptosis, or programmed cell death. If the change as a result of unweighting occurs within 12 24 hours, shared weight bearing may inhibit atrophic change efficiently.

The purpose of this study was to examine differences in the inhibitory effect of atrophy as a result of intermittent weight bearing in terms of the dynamics of nuclei in rat soleus muscle. In addition to histochemical examination of muscle fibers, this study immuno-histochemically examined both cell proliferation and apoptosis in the context of the dynamics of myonuclei.

\section{Methods}

\section{Experimental animals and design}

Forty-nine male Wistar rats (body wt: 190-228 g, age: 8 weeks) were used in this study. The animals were housed in individual cages at our facility under a 12-hour light-dark cycle (light: 8:00-20:00) and maintained on a diet of rodent chow and water ad libitum. At all times, the rats were treated in accordance with the guidelines for care and use of laboratory animals in Takara-machi Campus of Kanazawa University.

The rats were divided into a control group ( $\mathrm{CON}, \mathrm{n}=9)$ and an experimental group. The experimental group was subjected to hindlimb suspension for two weeks and subdivided into four groups: hindlimb suspension only (HS, $\mathrm{n}=11$ ), weight bearing for 10 minutes $\times 4$ times/day (at 8:00, 12:00, 16:00, and 19:50: W10, $\mathrm{n}=10$ ), weight bearing for 20 minutes $\times 2$ times/day (at 8:00 and 19:40: W20, $n=9$ ), and weight bearing for 40 minutes $\times$ one time/day (at 8:00: $\mathrm{W} 40, \mathrm{n}=10$ ). Hindlimb suspension was non-invasive, consisting of application of a simplified jacket as described in a previous study ${ }^{14) 15}$, so that the hindlimbs did not touch the floor. The hindlimb muscles were exposed to unweighting to induce disuse muscle atrophy. During suspension, the rats could move their forelimbs freely for the intake of chow and water. Weight bearing was induced by temporarily removing the suspension so that the rats were supporting their body weight on all four limbs, and took place during the light cycle when rats are relatively inactive. Total weight bearing time per day (40 minutes) in this study was designed according to the method of Widrick et al. ${ }^{10)}$ and Bangart et al. ${ }^{12)}$

\section{Injection of 5-bromo-2'-deoxyuridine (BrdU)}

Myonuclear dynamics in association with cell proliferation were examined by means of the nonradioactive BrdU technique ${ }^{22) 23}$ for labeling $S$-phase nuclei. Intraperitoneal injection ( $1 \mathrm{ml} / 100 \mathrm{~g}$ body $\mathrm{wt}$ ) of $\mathrm{BrdU}$ (Amersham, Buckinghamshire, UK) was administered $6 \mathrm{~h}$ before muscle extraction. Preliminary examination and previously reported results had indicated that proliferating myonuclei in the $S$ phase of the cell cycle within this interval would incorporate BrdU during DNA replication, and that non-replicating myonuclei, including the postmitotic mature myonuclei and quiescent satellite cells, would remain unlabeled.

\section{Muscle preparation}

At the end of the experimental period (two weeks), body weight was measured, the right soleus muscle was extracted under anesthesia (pentobarbital sodium, $50 \mathrm{mg} / \mathrm{kg}$ body wt, ip) and the muscle wet weight was measured. The muscles were then rapidly frozen in isopentane cooled by liquid nitrogen and stored at $-70^{\circ} \mathrm{C}$.

\section{Histochemical analysis}

Transverse sections (10 $\mu \mathrm{m}$ in thickness) were cut in a cryostat microtome at $-25^{\circ} \mathrm{C}$ and classified as to muscle fiber type (I and II) by means of adenosine triphosphatase (ATPase) staining (pH 10.6). The cross-sectional area (CSA) of more than 200 muscle fibers in each muscle, as the quantitative index of muscle atrophy, was measured as described in a previous study ${ }^{16)}$

\section{Immuno-cytochemical analysis}

Labeling of BrdU was used in an immunocytochemical system (Cell proliferation kit RPN20; Amersham) for monitoring cell proliferation by using monoclonal anti-BrdU. Transverse sections (5 $\mu \mathrm{m}$ in thickness) were cut and stained according to the assay procedure specified for the kit. The stained sections were examined by means of image analysis systems, each 
Table 1. Body and soleus muscle weights

\begin{tabular}{ccccc}
\hline Group & (n) & Body weight & Soleus wet weight & $\begin{array}{c}\text { Relative weight } \\
\text { Soleus wet weight }(\mathrm{mg}) \\
\text { /body weight }(\mathrm{g})\end{array}$ \\
\hline CON & $(9)$ & $304 \pm 23^{\dagger}$ & $140 \pm 36^{\dagger}$ & $0.45 \pm 0.09^{\dagger}$ \\
HS & $(11)$ & $203 \pm 15^{*}$ & $65 \pm 21^{*}$ & $0.32 \pm 0.10^{*}$ \\
W10 & $(10)$ & $196 \pm 14^{*}$ & $74 \pm 13^{*}$ & $0.38 \pm 0.06$ \\
W20 & $(9)$ & $194 \pm 14^{*}$ & $66 \pm 13^{*}$ & $0.34 \pm 0.06$ \\
W40 & $(10)$ & $194 \pm 12^{*}$ & $77 \pm 14^{*}$ & $0.39 \pm 0.06$ \\
\hline
\end{tabular}

${ }^{\dagger} \mathrm{p}<0.05$ (compared with HS), *: $\mathrm{p}<0.05$ (compared with CON). $\quad \mathrm{n}$ : No. of rats.

consisting of a light photomicroscope (BX-50; Olympus), personal computer (Power Macintosh G3), and image processing software (NIH Image 1.62). In four fields from each muscle, $430 \times 330 \mu \mathrm{m}$ each in size, the number of BrdU-labeled myonuclei and all fibers were counted. The labeled nuclei were classified as myonuclei (including satellite cells), except connective tissue nuclei, depending on their position relative to the fiber cross section. The number of BrdU-labeled myonuclei per 100 muscle fibers was revealed as the labeling index. The percentage of BrdU-labeled myonuclei (\%) was also expressed relative to the total number of hematoxylin-stained myonuclei per 100 muscle fibers.

Terminal deoxynucleotidyl transferase (TdT)-mediated dUTP nick end-labeling (TUNEL) ${ }^{24) 25)}$ was used for identification of apoptotic nuclei. The TUNEL method preferentially labels the 3'-OH ends exposed by doublestrand breaks in the DNA, the main form of cleavage during apoptosis, and has been used to label apoptotic nuclei in skeletal muscle. The labeling was performed with a commercial kit (Apoptosis in situ Detection Kit, Wako, Osaka, Japan) using the appropriate assay procedure. Analysis was performed in the same way for BrdU.

\section{Statistical analysis}

Data were expressed as means \pm SD. For statistical evaluation of differences among groups, a one-way analysis of variance (ANOVA) was performed, followed by Scheffe's post hoc test. An alpha level of 0.05 was set as the limit for statistical significance.

\section{Results}

\section{Body weight and muscle wet weight}

Two weeks of hindlimb suspension, simulating nonweight bearing or inactivity, produced a siginificant decrease in body weight. Compared with CON, the soleus muscle wet weight of the experimental group was significantly smaller. No statistically significant difference among the experimental groups was observed in either body weight or muscle wet weight. The muscle-to-body weight (relative weight) was the highest for CON (0.45) and the lowest for HS (0.32). The ratios for the experimental groups with intermittent weight bearing (weight bearing groups) were not significantly different from those for CON and HS (Table 1).

\section{Fiber CSA of soleus mucle}

The mean CSA of type I and II fibers in CON was significantly larger than that of the experimental groups (Fig. 1). The mean CSA of type I fibers of HS (1464 \pm 592 $\left.\mu \mathrm{m}^{2}\right)$ decreased to $50.2 \%$, of W10 (2082 $\left.\pm 712 \mu \mathrm{m}^{2}\right)$ to $71.4 \%$, of W20 (1622 $\left.\pm 492 \mu \mathrm{m}^{2}\right)$ to $55.6 \%$, and of $\mathrm{W} 40$ $\left(1837 \pm 630 \mu \mathrm{m}^{2}\right)$ to $63.0 \%$ of the values for CON (2916 \pm $1054 \mu \mathrm{m}^{2}$ ) (Fig. 2). Statistically significant differences were observed among the weight bearing groups (W10 > $\mathrm{W} 40>\mathrm{W} 20)$. The mean CSA of type II fibers of HS (1041 $\left.\pm 417 \mu \mathrm{m}^{2}\right)$ decreased to $45.1 \%$, of W10 (1393 $\left.\pm 505 \mu \mathrm{m}^{2}\right)$ to $60.3 \%$, of W20 $\left(1230 \pm 351 \mu \mathrm{m}^{2}\right)$ to $53.2 \%$, and of W40 $\left(1334 \pm 343 \mu \mathrm{m}^{2}\right)$ to $57.7 \%$ of the values for CON (2310 \pm $579 \mu \mathrm{m}^{2}$ ). Statistically significant differences were observed among the weight bearing groups, except between W10 and W40 (Fig. 2).

\section{Number of myonuclei}

No statistically significant difference among all groups was observed in the mean number of myonuclei per muscle fiber in transverse section (CON: $2.3 \pm 0.5$, HS: $2.1 \pm 0.3$, W10: $2.2 \pm 0.4$, W20: $1.8 \pm 0.5$, W40: $2.3 \pm 0.3$ ).

\section{Labeling of $\mathrm{BrdU}$}

Labeled myonuclei localized within the peripheral areas of fibers, stained dark brown (Fig. 3-A, B). The labeling index was significantly lower for the experimental groups than for $\operatorname{CON}(5.7 \pm 0.9,2.5 \%)$. Among the experimental groups, a statistically significant difference was recognized between any two groups, except between HS $(1.4 \pm 0.5,0.7 \%)$ and W20 $(2.0 \pm 0.4,1.1 \%)$ or between $\mathrm{W} 10(2.8 \pm 0.5,1.3 \%)$ and W20. Among the weight bearing groups, the labeling index of both W10 and W40 

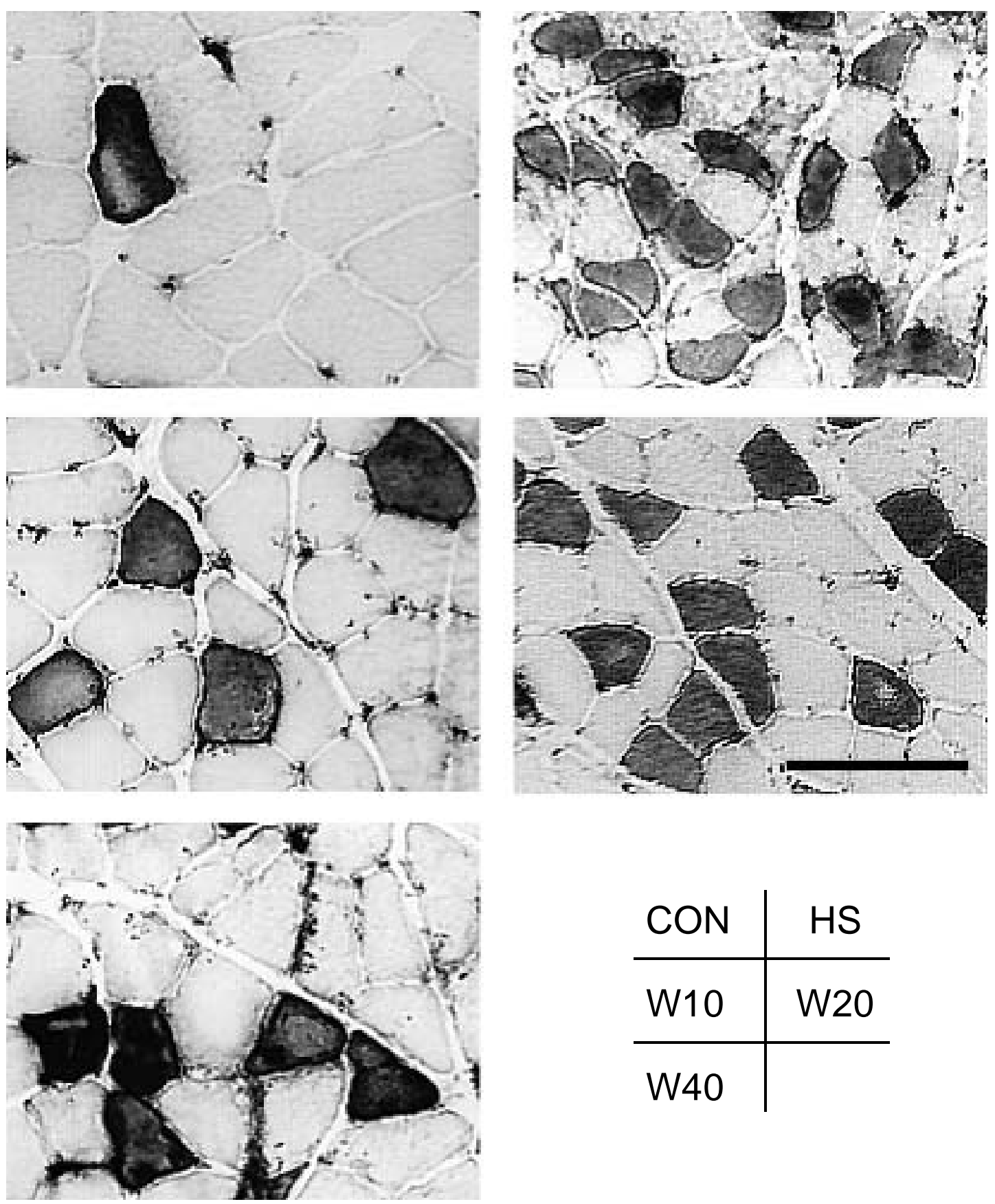

Fig. 1. Photomicrograph of soleus muscle (cross section). ATPase staining was used to discriminate type I (light stain) and type II (dark stain) fibers. The scale bar represents $100 \mu \mathrm{m}$.

$(4.3 \pm 1.0,1.9 \%)$ was significantly higher than $\mathrm{HS}$, and that of W40 was significantly higher than W10 (Fig. 4).

\section{Labeling used TUNEL}

Labeled myonuclei localized within the peripheral areas of fibers, also stained dark brown (Fig. 3-C, D). The labeling index was significantly higher for W20 (4.6 \pm 2.0 , $2.6 \%)$ and HS $(6.0 \pm 1.9,2.9 \%)$ than for CON $(0.4 \pm 0.4$, $0.2 \%)$. Among the experimental groups, the labeling index of W10 $(2.3 \pm 1.0,1.0 \%)$ and W40 $(2.4 \pm 1.3,1.0 \%)$ was significantly lower than HS. No statistically significant differences in the labeling index were observed between HS and W20 or between W10 and W40 (Fig 5).

\section{Discussion}

Atrophy at the whole muscle level was assessed by means of muscle wet weight and relative weight. Soleus wet weight of the experimental group was smaller than that of CON, and showed marked atrophy as a result of hindlimb suspension (unweighting). No difference in relative weight was observed between the weight bearing groups and CON, 


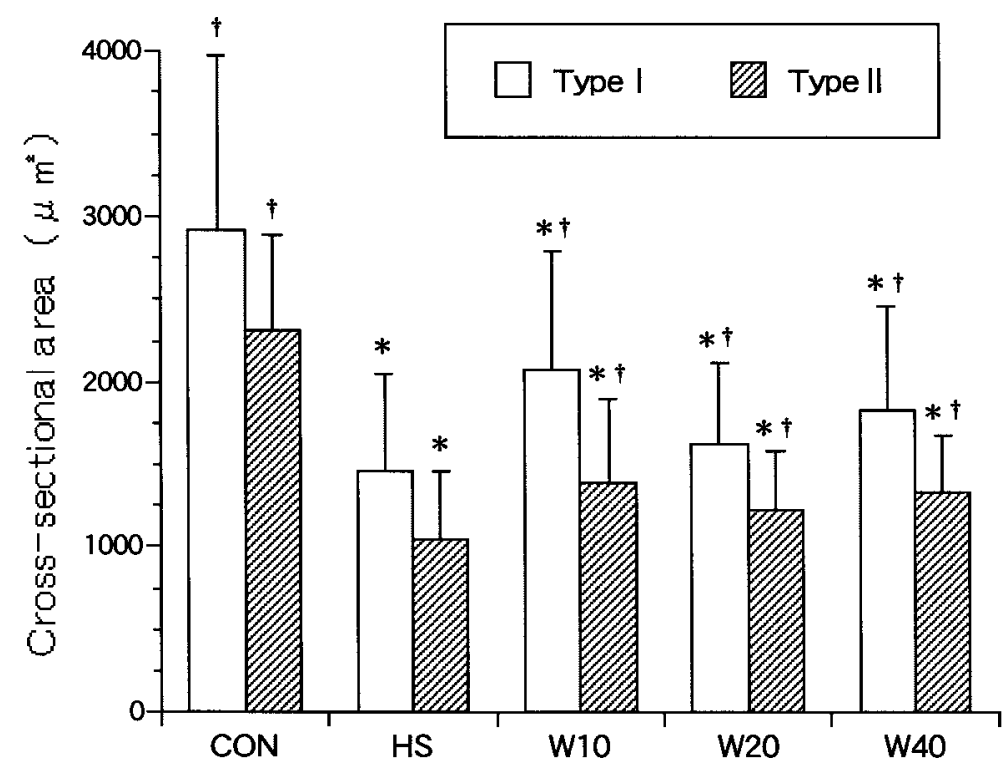

Fig. 2. Cross-sectional area of soleus muscle fibers. ${ }^{\dagger}: \mathrm{p}<0.05$ (compared with HS), *; $\mathrm{p}<0.05$ (compared with $\mathrm{CON}$ ). Values are means \pm SD.
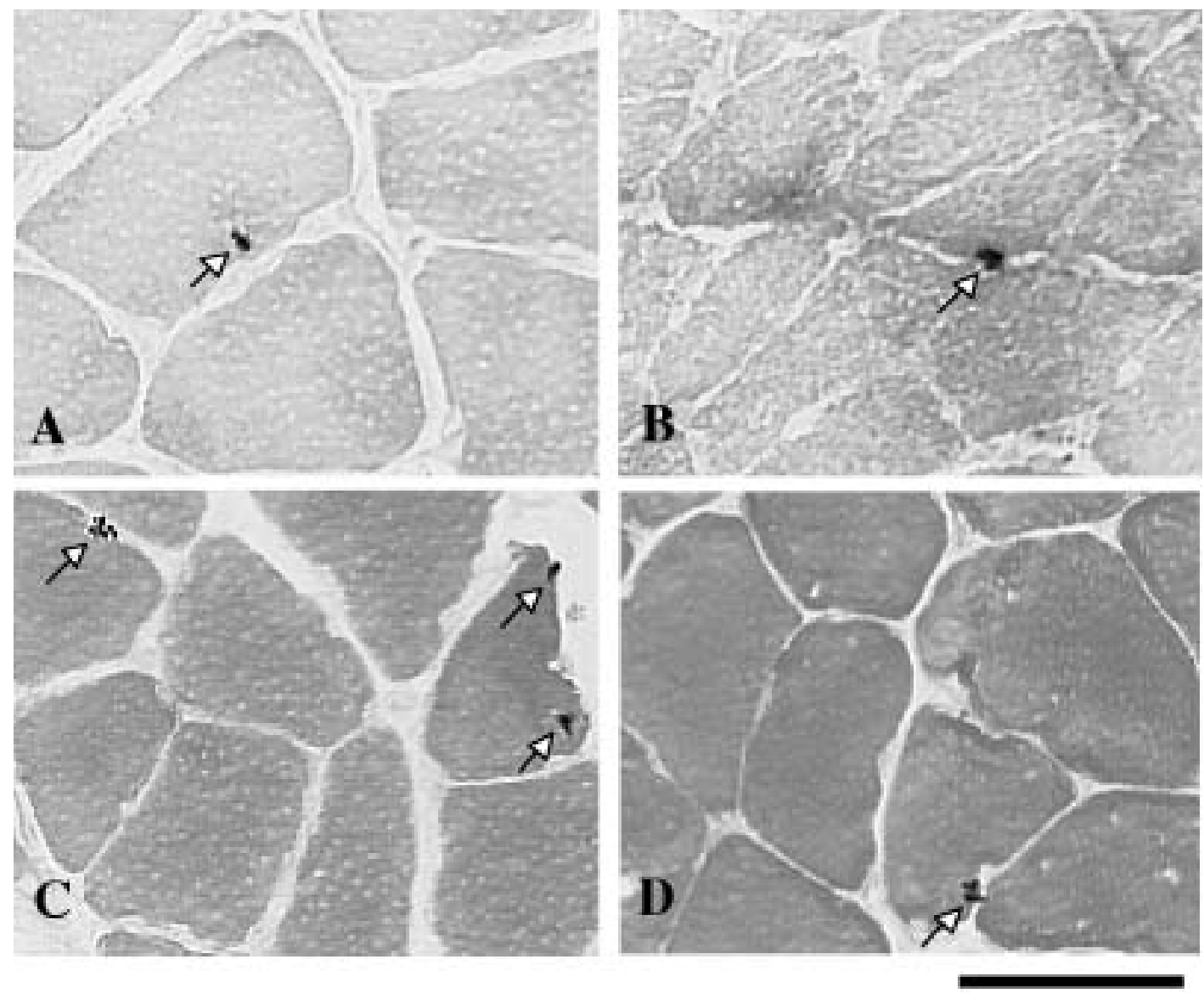

Fig. 3. Photomicrograph of labeled myonuclei (arrows) immunohistochemically. Croos-section counterstained with eosin. The scale bar represents $50 \mu \mathrm{m}$. A: Labeling of BrdU (CON). B: Labeling of BrdU (W10). C: Labeling used TUNEL (W20). D: Labeling used TUNEL (W40). 


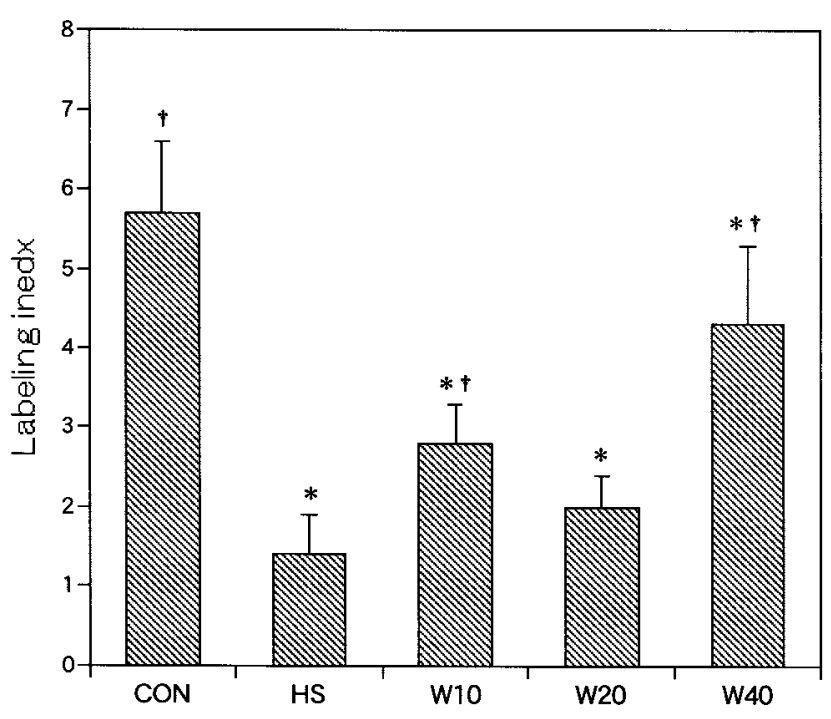

Fig. 4. The number of BrdU-labeled myonuclei. Labeling index: labeled myonuclei/100 soleus muscle fibers. †: $\mathrm{p}<0.05$ (compared with HS), *: p<0.05 (compared with $\mathrm{CON})$.

thus demonstrating the effect of weight bearing. The results for fiber CSA clearly showed the effect, too, although fiber CSA of the weight bearing group was somewhat smaller than that of CON. These findings indicate that the weight bearing method used in this study could not completely prevent disuse muscle atrophy, but could suppress its progression.

Recent studies have demonstrated that muscle atrophy is associated with a decrease in myonuclear number. Allen et al. ${ }^{18)}$ reported that the mean myonuclear number of fibers expressing type I myosin heavy chain decreased after 2 weeks of suspension compared with control in longitudinal section (134 vs. 162 myonuclei/mm). In this study, however, myonuclear number in transverse section did not change significantly. After this, it will be necessary to examine in longitudinal section.

As to cell proliferation, Smith et al. ${ }^{23)}$ reported that the percentage of fibers with BrdU-labeled proliferating myonuclei was low in normal rat $(10 \sim 12 \mathrm{w})$. It is apparent from this low rate of proliferation that some cells are going through the replicative cell cycle, even in the absence of an externally applied stimulus such as exercise or injury. The proliferation rate in normal rat may indicate fiber turnover in this postural muscle. The BrdU-labeling index for the experimental group was significantly lower than for $\mathrm{CON}$, indicating suppression of proliferation as a result of hindlimb suspension. In the experimental group, the BrdUlabeling index for W10 and W40 was higher than for HS, demonstrating the effect of weight bearing. However, W20 was ineffective in term of weight bearing. Furthermore, the index for W40 was significantly higher than that for W10, suggesting a different effect by different weight bearing

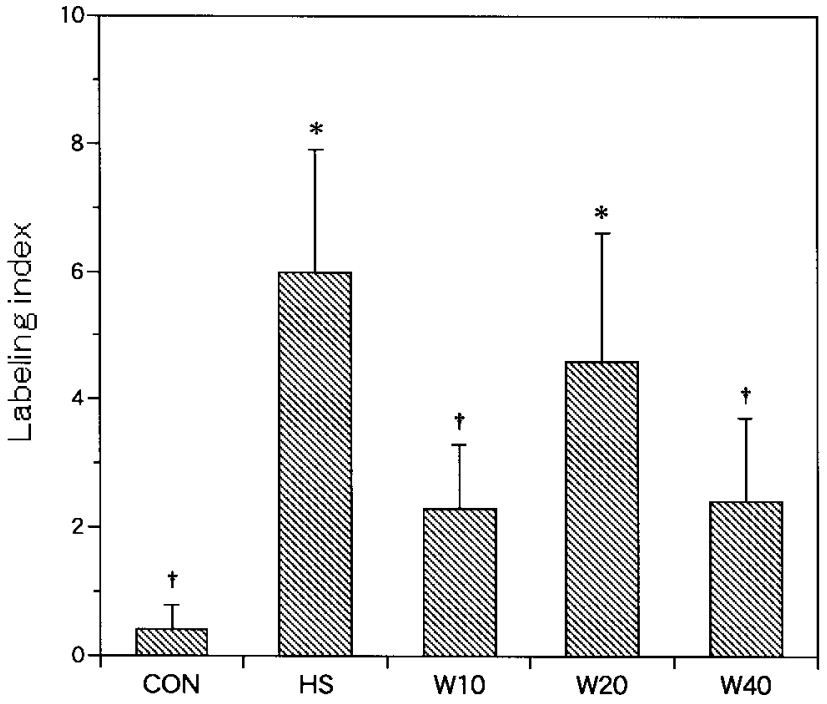

Fig. 5. The number of myonuclei labeled with TUNEL. Labeling index: labeled myonuclei/100 soleus muscle fibers. ${ }^{\dagger}: \mathrm{p}<0.05$ (compared with HS), *: $\mathrm{p}<0.05$ (compared with CON).

methods on proliferation. Because weight bearing covered the same amount of time per day (40 minutes in this study), it indicates that weight bearing once a day has a greater effect on proliferation than weight bearing spread over four times. However, the ineffectiveness of W20 suggests the influence of other factors than weight bearing frequency. For type I fiber CSA, W10 was more effective than W40, demonstrating the opposite result of that for the BrdUlabeling index. To account for this discrepancy, it is necessary to consider the time course. Schultz et al. ${ }^{20)}$ reported that the mitotic activity of satellite cells is a sensitive indicator of primary atrophic changes occurring in growing myofibers, and may be a predictor of future morphological changes.

The mechanism(s) responsible for the loss of myonuclei during conditions of muscle atrophy are currently not clearly understood, nor is it clear how individual myonuclei can be eliminated without destruction of all myonuclei or the entire myofiber ${ }^{18)}$. Several possible explanations can be envisioned. First, it is possible that reduced neuromuscular activity results in the degeneration of all fibers, which are then replaced following satellite cell proliferation and fusion. A second possibility is that there is preferential elimination of fibers containing large numbers of myonuclei. A third possibility is that reduced neuromuscular activity results in a depression of normal satellite cell proliferative activity. This appears to be the mechanism by which the number of myonuclei is attenuated during hindlimb suspension in young animals ${ }^{26)}$. A more likely possibility, however, is that myonuclei are eliminated from atrophying adult myofibers by apoptosis. This genemediated process (apoptosis) contributes to pre- 
programmed nuclear and cellular elimination during the development and regression of many biological structures and tissues, including skeletal muscle. In adult muscle, the presence of apoptotic myonuclei has been reported during the inactivity and atrophy induced by hindlimb suspension $^{18)}$. On the basis of results for CON in this study, we believe that apoptosis is extremely slight in normal muscle. Allen et al. ${ }^{27)}$ also reported that normal turnover of myonuclei was low in the soleus of control rats. The TUNEL-labeling index for HS was significantly higher than for CON. This suggests that elimination of existing myonuclei by apoptosis is at least partly responsible for the reduction in the number of myonuclei accompanying hindlimb suspension-induced atrophy. With the weight bearing method used for this study, W20 was not significantly different from HS in terms of apoptosis and was ineffective as weight bearing. W10 and W40, on the other hand, were not significantly different from CON, suggesting that these weight bearing methods can prevent the loss of myonuclei by apoptosis.

Some other reports have been published on the effect of intermittent weight bearing during hindlimb suspension ${ }^{5-12)}$. The results of these reports largely agree where partial suppression is concerned (not complete prevention), but vary in terms of the degree of the progression of muscle atrophy. The weight bearing protocol performed in this study, a modification of the method of Widrick et al. ${ }^{10)}$ and Bangart et $a l .{ }^{12)}$, produced results supported by those of previous studies. Brown et al. ${ }^{9)}$ described the effect of weight bearing for $1 \mathrm{~h} /$ day from the day following the start of suspension, and suggested that this treatment was effective. We also investigated the effect of intermittent weight bearing at varying intervals, and found that weight bearing at intervals of 12 and 24 hours was effective, but ineffective at intervals of 4 and 8 hours for suppression of the progression of muscle atrophy ${ }^{16)}$. It was thus suggested that the weight bearing interval is an important factor. In case of re-weight bearing after hindlimb suspension for 12.5 days, Krippendorf et al. ${ }^{3)}$ reported that 12 48 hours of re-weight bearing induced macrophage activation. Schultz et $a l .{ }^{20)}$ reported that suppression of mitotic activity decreased with 12 24 hour intervals, but it did not decrease for interval of less than 12 hours after initiation of hindlimb suspension. In a previous study of intermittent weight bearing frequency in a week, daily weight bearing was more effective compared with weight bearing every other day ${ }^{13)}{ }^{14)}$. W20 in this study had no effect on the dynamics of myonuclei, while weight bearing at intervals of 12 hours is clinically difficult to implement. We therefore conclude that weight bearing at intervals of 24 hours, that is once per day, is most suitable. However, there are many clinical cases in which it is difficult to impose weight bearing for a long time. For that reason, this study investigated the different inhibitory effects on atrophy by different methods of intermittent weight bearing while keeping the total daily weight bearing time constant. The results in this study indicate the possibility of inducing different effects by the frequency of weight bearing. Weight bearing spread over two times (W20) was not so effective, but weight bearing once (W40) or spread over four times (W10) was effective as weight bearing. Also, weight bearing once was more effective for cell proliferation than spread over four times. It will be necessary to examine the interaction of other factors besides the number of weight bearing sessions.

\section{Acknowledgement}

The author would like to thank Prof. Nobuhide Haida, School of Health Sciences, Faculty of Medicine, Kanazawa University, for his advice.

\section{References}

1) St-Pierre D, Leonard D, et al.: Recovery of muscle from tetrodotoxin-induced disuse and the influence of daily exercise. 1. Contractile properties. Exp Neurol 98: 472-488, 1987.

2) Yamazaki $T$, Tachino $K$, et al.: Effect of weight-bearing on disuse muscle atrophy in rats. Study of weight-bearing time in a day. Memoirs Al Med Prof Kanazawa Univ 17: 63-67, 1993.

3) Krippendorf BB, Riley DA: Distinguishing unloading- versus reloading-induced changes in rat soleus muscle. Muscle Nerve 16: 99-108, 1993.

4) St-Pierre BA, Tidaball JG: Differential response of macrophage subpopulations to soleus muscle reloading after rat hindlimb suspension. J Appl Physiol 77: 290-297, 1994.

5) Herbert ME, Roy RR, et al.: Influence of one-week hindlimb suspension and intermittent high load exercise on rat muscles. Exp Neurol 102: 190-198, 1988.

6) Thomason DB, Herrick RE, et al:: Activity influences on soleus muscle myosin during rodent hindlimb suspension. $\mathbf{J}$ Appl Physiol 63: 138-144, 1987.

7) D'Aunno DS, Thomason DB, et al.: Centrifugal intensity and duration as countermeasures to soleus muscle atrophy. J Appl Physiol 69: 1387-1389, 1990.

8) D'Aunno DS, Robinson RR, et al.: Intermittent acceleration as a countermeasure to soleus muscle atrophy. J Appl Physiol 72: 428-433, 1992.

9) Brown M, Hasser EM: Weight-bearing effects on skeletal muscle during and after simulated bed rest. Arch Phys Med Rehabil 76: 541-546, 1995.

10) Widrick JJ, Bangart JJ, et al.: Soleus fiber force and maximal shortening velocity after non-weight bearing with intermittent activity. J Appl Physiol 80: 981-987, 1996.

11) Alley KA, Thompson LV: Influence of simulated bed rest and intermittent weight bearing on single skeletal muscle fiber function in aged rats. Arch Phys Med Rehabil 78: 19-25, 1997.

12) Bangart JJ, Widrick JJ, et al.: Effect of intermittent weight bearing on soleus fiber force-velocity-power and force-pCa 
relationships. J Appl Physiol 82: 1905-1910, 1997.

13) Yamazaki $T$, Haida $N$, et al.: Effect of weight-bearing in prevention of disuse atrophy in rat hindlimb muscles. Study of weight-bearing frequency in a week. Rigaku ryohogaku 22: 108-113, 1995 (In Japanese with English abstract).

14) Yamazaki $\mathrm{T}$, Haida $\mathrm{N}$, et al.: Effect of weight-bearing frequency per day in retarding disuse atrophy in rat soleus muscle. Rigaku ryoho janaru 30: 53-57, 1996.

15) Yamazaki $\mathrm{T}$ : Effect of weight-bearing on disuse muscle atrophy in rats. Rigaku ryohogaku 23: 417-420, 1996 (In Japanese with English abstract).

16) Yamazaki $T$, Haida $N$, et al.: Effects of weight bearing intervals on disuse atrophy of rat soleus muscle. J Jpn Phys Ther Assoc 1: 19-24, 1998.

17) Yamazaki $T$, Haida $N$, et al:: Influence of the time when weight bearing is started on disuse atrophy in rat soleus muscle. J Jpn Phys Ther Assoc 4: 13-18, 2001.

18) Allen DL, Roy RR, et al:: Myonuclear domains in muscle adaptation and disease. Muscle Nerve 22: 1350-1360, 1999.

19) Allen DL, Monke SR, et al.: Plasticity of myonuclear number in hypertrophied and atrophied mammalian skeletal muscle fibers. J Appl Physiol 78: 1969-1976, 1995.

20) Schultz E, Darr KC, et al.: Acute effects of hindlimb unweighting on satellite cells of growing skeletal muscle. J
Appl Physiol 76: 266-270, 1994.

21) Allen DL, Yasui W, et al.: Myonuclear number and myosin heavy chain expression in rat soleus single muscle fibers after spaceflight. J Appl Physiol 81: 145-151, 1996.

22) Schmalbruch H, Lewis DM: Dynamics of nuclei of muscle fibers and connective tissue cells in normal and denervated rat muscles. Muscle Nerve 23: 617-626, 2000.

23) Smith HK, Maxwell L, et al.: Exercise-enhanced satellite cell proliferation and new myonuclear accretion in rat skeletal muscle. J Appl Physiol 90: 1407-1414, 2001.

24) Smith HK, Maxwell L, et al: Nuclear DNA fragmentation and morphological alterations in adult rabbit skeletal muscle after short-term immobilization. Cell Tissue Res 302: 235241, 2000.

25) Olive M, Ferrer I: Bcl-2 and Bax immunohistochemistry in denervation-reinnervation and necrosis-regeneration of rat skeletal muscles. Muscle Nerve 23: 1862-1867, 2000.

26) Darr KC, Schultz E: Hindlimb suspension suppresses muscle growth and satellite cell proliferation. J Appl Physiol 67: 1827-1834, 1989.

27) Allen DL, Linderman JK, et al:: Apoptosis: a mechanism contributing to remodeling of skeletal muscle in response to hindlimb unweighting. Am J Physiol 273: 579-587, 1997. 